\title{
Reeducação Funcional da Pessoa Com Deglutição Comprometida: Estudo De Caso
}

\section{ReEducación Funcional De La Persona Con Deglución Comprometida: Estudio De Caso functional Reeducation Of Person With Committed Deglutition: Case Study}

\author{
Paulo César Lopes Silva ${ }^{1}$; Eugénia Nunes Grilo² \\ 1 - Unidade Local de Saúde do Baixo Alentejo, EPE; \\ 2 - Escola Superior de Saúde Dr. Lopes Dias, Instituto Politécnico de Castelo Branco
}

\section{RESUMO}

Objetivos: Identificar os ganhos sensíveis aos cuidados de enfermagem de reabilitação com um programa de optimização da deglutição numa pessoa com deglutição comprometida.

Método: Estudo de abordagem qualitativa, tipo estudo de caso. Centra-se na aplicação do Processo de Enfermagem, respeitando a linguagem CIPE $^{\circledR}$, e recorre ao Padrão Documental dos Cuidados da Especialidade de Enfermagem de Reabilitação para a fundamentação da intervenções implementadas. Expõe-se o caso de uma pessoa com deglutição comprometida, de etiologia neurológica, admitida numa Unidade de Internamento de um Centro Hospitalar, sendo assegurados os principios éticos na sua abordagem.

Resultados: Não foram evidenciadas complicações na implementação do programa e observou-se a recuperação completa da função deglutição, após dez sessões de treino.

Conclusões: A sistematização dos cuidados de enfermagem de reabilitação revelou-se eficaz para reverter a alteração observada e concorreu para incrementar a autonomia da pessoa.

Descritores: Transtornos de Deglutição, Relatos de Casos, Enfermagem em Reabilitação; Autocuidado.

\section{RESUMEN}

Objetivos: Identificar las ganancias sensibles a los cuidados de enfermería de rehabilitación con un programa de optimización de la deglución en una persona con deglución comprometida.

Metodología: Estudio de enfoque cualitativo, tipo estudio de caso. Se centra en la aplicación del Proceso de Enfermería, respetando el lenguaje CIPE ${ }^{\oplus}$, y recurre al "Pátron Documental de los Cuidados Especializados de la Especialidad de Enfermería de Rehabilitación" para la fundamentación de las intervenciones implementadas. Se expone el caso de una persona con deglución comprometida, de etiología neurológica, admitida en una Unidad de un Centro Hospitalario, siendo asegurados los principios éticos en su abordaje.

Resultados: No se evidenciaron complicaciones en la implementación del programa y se observó la recuperación completa de la función deglución, después de diez sesiones de entrenamiento.

Conclusiones: La sistematización de los cuidados de enfermería de rehabilitación resultó eficaz para revertir la alteración observada y permitió incrementar la autonomía de la persona.

Descriptores: Trastornos de Deglución, Informes de Casos, Enfermeria en Rehabilitación, Autocuidado.

\section{ABSTRACT}

Objectives: To identify the sensible gains for rehabilitation nursing care with a swallow optimization program in a person with compromised swallowing.

Methods: Qualitative study, type of case study. It focuses on the application of the Nursing Process, respecting the CIPE $^{\circledR}$ language, and uses the Documentary Pattern of Care of the Rehabilitation Nursing Specialty for the substantiation of the implemented interventions. We present the case of a person with compromised swallowing, of neurological etiology, admitted to a Hospital Center inpatient unit, assuring the ethical principles in their approach.

Results: There was no evidence of complications in the implementation of the program and complete recovery of the swallowing function was observed after ten training sessions.

Conclusion: The systematization of rehabilitation nursing care proved to be effective in reversing the observed change and contributed to increase the person's autonomy

Descriptors: Deglutition disorders, Case Reports, Rehabilitation nursing, Self Care. 


\section{INTRODUÇÃO}

$\mathrm{Na}$ abordagem à pessoa com deglutição comprometida é importante que a equipa multidisciplinar se encontre consciente das suas consequências e saiba identificar os seus sinais $\operatorname{clínicos}^{(1)}$. Assim, uma avaliação precoce da função de deglutir é tão ou mais importante quanto a avaliação do estado de consciência da pessoa e a equipa de enfermagem deve estar preparada para a rápida identificação destas pessoas, facilitando o diagnóstico e o tratamento da alteração em causa ${ }^{(2)}$.

A intervenção do enfermeiro, nomeadamente do enfermeiro de reabilitação é fundamental junto das pessoas com deglutição comprometida, acompanhandoas no processo de reabilitação desde o seu início e em permanência constante.

A percepção da pessoa é igualmente fundamental no diagnóstico de enfermagem "deglutição comprometida". Nesse sentido, o Eat Assessement Tool (EAT-10) é um instrumento passível de ser aplicado na pessoa com deglutição comprometida, independentemente do seu diagnóstico, pois permite mensurar o grau de alteração da deglutição sentido pela mesma $^{(3)}$.

Trata-se de um questionário do tipo Likert onde a pessoa refere a intensidade da alteração percepcionada, atribuindo uma pontuação a cada uma das dez afirmações, a partir das quais se obtém um score que pode variar entre 0 para nenhum problema e 4 que corresponde a problema grave. Uma pontuação final superior ou igual a três pontos orienta para a presença ou risco de deglutição comprometida ${ }^{(3)}$.

Um estudo indica que $48 \%$ das pessoas com aspirações detetadas por videofluroscopia não apresentaram tosse após aspiração de conteúdo deglutido, atestando uma elevada incidência de aspirações silenciosas ${ }^{(4)}$. Tendo em conta esta problemática, o Volume-Viscosity Swallow Test (V-VST) é um método de exploração clínico, de fácil aplicação, que recorre à oximetria de pulso como procedimento complementar para a rápida identificação dessas pessoas sem recursos a métodos invasivos como a videofluroscopia ou videoendoscopia ${ }^{(4)}$.

O referido método permite a rápida deteção de sinais clínicos de alteração na eficácia e segurança da deglutição através da administração de três tipos de consistências: néctar, líquido e pudim, respeitando um fluxograma com diversos graus de dificuldade ${ }^{(4)}$. As alterações na eficácia compreendem: o encerramento labial ineficaz, a presença de resíduos orais ou faríngeos e as múltiplas deglutições por bolo alimentar administrado. Os sinais de alteração na segurança compreendem: a alteração na qualidade da voz, a tosse ou a diminuição da oximetria de pulso superior ou igual a $3 \%(4)$.

Com os dados obtidos na aplicação do método V-VST é possivel a categorização das limitações funcionais na alimentação por via oral(4). Neste sentido, a Functional Oral Intake Scale (FOIS), com os seus 7 itens, é um instrumento de avaliação que permite essa abordagem. Uma pessoa posicionada no nível 7 não apresenta qualquer tipo de limitação, por sua vez, uma pessoa posicionada no nível 1 não é capaz de deglutir qualquer alimento por via oral ${ }^{(5)}$.

Por sua vez, nos níveis 1 a 3 deste instrumento são posicionados aqueles que necessitam de sonda nasogástrica para a manutenção de um aporte alimentar ou hídrico adequado. Os níveis 4 a 7 encontram-se destinados para aqueles que apresentam capacidade para a ingestão por via oral sem necessitar de qualquer via alternativa ${ }^{(5)}$.

O EAT-10 e a FOIS apresentam-se como dois instrumentos de fácil aplicação( ${ }^{(5)}$, encontram-se validados para a população portuguesa ${ }^{(3 ; 5)}$ e constituem uma mais-valia para a avaliação das pessoas com deglutição comprometida, orientando a sua respetiva reabilitação( ${ }^{(5)}$.

No tratamento das alterações da deglutição é importante a identificação precisa das estruturas afetadas e do tipo de défice na deglutição. Se for identificada fraqueza muscular, a pessoa deve ser orientada para a execução de uma série de exercícios centrados na reeducação de áreas específicas da base da língua ou das paredes laterais da faringe. No caso da presença de défice neurológico que curse em alterações no reflexo de deglutição ou se o défice muscular for inultrapassável, então a pessoa deve ser orientada para a execução de estratégias compensatórias para melhorar a segurança da deglutição(6).

Após a identificação da alteração da deglutição, tornase fundamental delinear o planeamento dos cuidados de enfermagem, almejando a reeducação funcional da deglutição que, dada a sua complexidade, requer competências específicas no campo de intervenção ${ }^{(7)}$.

0 processo de reabilitação da deglutição comprometida engloba, desta forma, componentes de treino de habilidades e de força muscular, que visam assegurar uma ingestão oral segura e combater as alterações da deglutição( ${ }^{(8)}$.

Em paralelo, também é fundamental implementar outros cuidados de enfermagem de reabilitação, tais como técnicas de melhoria do padrão ventilatório e mecânica ventilatória, procurando assegurar a permeabilidade das vias aéreas, promover o fortalecimento da musculatura respiratória e reduzir o risco de aspiração ${ }^{(7)}$.

A prevenção das consequências da imobilidade deve incluir intervenções de enfermagem centradas na promoção do autocuidado com recurso a estratégias adaptativas $^{(9)}$. É frequente observar-se alteração na mobilidade na pessoa com deglutição comprometida, tal alteração não pode ser descurada no planeamento e implementação de um programa de enfermagem de reabilitação. Desta forma, as intervenções de enfermagem, neste âmbito, devem ser promotoras do autocontrolo e autocuidado destas pessoas.

Apresentando-se a reabilitação como um processo que ambiciona a recuperação funcional da pessoa, a reintegração na família, na comunidade e na sociedade, torna-se necessária uma abordagem holística da pessoa 


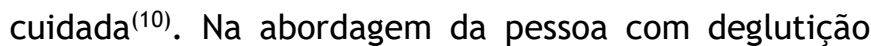
comprometida estas premissas devem ser sempre uma constante.

A Enfermagem de Reabilitação poderá desempenhar um papel fundamental na abordagem da pessoa com a referida disfunção. Esta especialidade, com outra área da Enfermagem, deve ser suportada num referencial que norteie a prática. Na presente abordagem, a teoria de Enfermagem do Défice de Autocuidado de Dorothea Orem encaixa-se na perfeição.

A referida teoria de enfermagem propõe cinco áreas de atividades para a prática, que são: a manutenção de uma relação terapêutica; a determinação de como a pessoa pode ser ajudada através dos cuidados de enfermagem; a resposta às necessidades da pessoa em relação ao contacto e à assistência do enfermeiro; a prescrição, fornecimento e regulação da ajuda direta à pessoa e conviventes significativos; a coordenação e integração dos cuidados de enfermagem na vida diária da pessoa; outra assistência de saúde ou os serviços sociais e de educação necessários ${ }^{(11)}$. As cinco áreas referidas encontram-se bem presentes ao longo do caso apresentado.

O presente estudo centrou-se na implementação de um programa de reeducação funcional, composto por intervenções de enfermagem de reabilitação junto de uma pessoa com deglutição comprometida de etiologia neurológica. Assim, foram desenvolvidas estratégias devidamente fundamentadas no Processo de Enfermagem e no Padrão Documental dos Cuidados Especializados da Especialidade de Enfermagem de Reabilitação(12). Igualmente, o uso de instrumentos de avaliação apropriados permitiu criar critérios de diagnóstico que ajudaram a definir diagnósticos de enfermagem de reabilitação mais acurados neste âmbito e procurou nortear o processo de avaliação dos resultados desse mesmo programa.

Neste sentido, o objetivo geral deste estudo foi identificar os ganhos sensíveis aos cuidados de enfermagem de reabilitação com a implementação de um programa de optimização da deglutição numa pessoa com deglutição comprometida. Os objetivos específicos visavam identificar as alterações da deglutição com recurso a três instrumentos avaliação de alterações da deglutição; definir os principais diagnósticos de enfermagem de reabilitação no âmbito da deglutição comprometida; avaliar o impacto da implementação de um programa de optimização da deglutição numa pessoa com deglutição comprometida, na melhoria dos diagnósticos de enfermagem de reabilitação.

\section{MATERIAL E MÉTODOS}

Em Enfermagem, a abordagem através do estudo de caso, permite ao investigador estudar um fenómeno complexo da vida de uma forma intensiva e profunda, recorrendo a múltiplas fontes de evidência ${ }^{(13)}$.

Os estudos de casos podem gerar hipóteses para futuros estudos clínicos, assim como orientam para a individualização e personalização da prestação de cuidados. Foram utilizadas as guidelines da CAse REport
(CARE) pois fornecem a estrutura necessária para satisfazer a precisão, integridade e transparência na abordagem científica de estudos de caso ${ }^{(14)}$.

As guidelines da CARE integram uma lista de verificação de 13 itens (título, palavras-chave, resumo, introdução, informação da pessoa, achados clínicos, cronograma, avaliação diagnóstica, intervenção terapêutica, seguimento clínico e resultados, discussão, perspetiva da pessoa, termo de consentimento informado) e encontra-se especialmente estruturada para corresponder aos principais componentes de um estudo de caso e apreender as informações clínicas de relevo ${ }^{(14)}$.

De igual forma, na estruturação do presente estudo foram cumpridas as seis etapas do desenvolvimento do estudo de caso definidas por Yin e Stake, são elas: Definição da problemática; Definição do caso; Fundamentação Teórica; Elaboração do protocolo do estudo; Colheita de dados; Análise e Interpretação dos resultados $^{(13)}$.

O presente estudo descreve o caso de uma pessoa com deglutição comprometida e apresenta caráter longitudinal, o que permite demonstrar o programa de reeducação funcional da deglutição desenvolvido junto dessa pessoa e evidenciar os resultados alcançados.

Foi realizado durante o mês de novembro de 2017, desde a admissão num serviço do departamento de medicina de um Centro Hospitalar da Administração Regional de Saúde de Lisboa e Vale do Tejo, até à recuperação funcional da deglutição. Trata-se de um caso único e de abordagem holística.

A pessoa envolvida no estudo assinou o formulário de consentimento informado esclarecido e livre para atos/intervenções de saúde nos termos da norma $n .^{\circ}$ 015/2013 da Direção-Geral da Saúde.

O estudo foi aprovado pela Comissão de Ética para a Saúde e autorizado pelo Conselho de Administração do Centro Hospitalar.

Vale ressaltar que foram respeitados todos os aspectos éticos interligados com a investigação realizada com seres humanos. Assim, procurou-se cumprir as indicações expressas na Declaração de Helsínquia, na Convenção sobre os Direitos do Homem e da Biomedicina, nas orientações do Council for International Organizations of Medical Sciences e no Guia das Boas Práticas Clínicas.

A pessoa foi designada por uma letra (A), salvaguardando a confidencialidade dos dados e o anonimato da mesma.

O recurso a instrumentos específicos para a avaliação do compromisso da deglutição requereu o pedido de autorização a investigadores nacionais que adaptaram para o português europeu esses mesmos instrumentos.

Este estudo evidencia os resultados de um participante que integrou um amplo projeto cujos critérios de inclusão dos participantes foram: pontuação na Escala de Comas de Glasgow superior ou igual a 11 pontos (pontuação máxima nos itens "abertura ocular" e "melhor resposta motora"), de forma a incluir as pessoas com défice de expressão; pontuação no EAT-10 
superior ou igual a 3 pontos (sugerindo a perceção de compromisso na deglutição $\left.{ }^{(3)}\right)$; FOIS superior a nível 1 e inferior a nível 7 (permitindo a inclusão de pessoas com via oral, mas com compromisso na ingestão de determinadas consistências ${ }^{(5)}$ ).

Foi realizada uma análise estatística de todos os questionários EAT-10 aplicados à pessoa ao longo do estudo, recorrendo ao programa informático IBM-SPSS (Statistical Package for the Social Sciences) versão 22.0, facilitando uma posterior análise descritiva dos mesmos.

\section{APRESENTAÇÃO DO CASO}

\section{Anamnese}

A anamnese orienta o profissional de saúde para o diagnóstico e respetivo plano terapêutico. As características socioeconómicas, a história pregressa, a história familiar e a história clínica atual são componentes fundamentais de uma boa anamnese ${ }^{(15)}$.

É exposto o caso do Sr. A de 57 anos de idade, género masculino, caucasiano e de nacionalidade portuguesa. Tem $09^{\circ}$ ano de escolaridade. É casado e não tem filhos. Quanto à sua situação laboral, trabalha numa empresa como técnico de elevadores. Foi admitido no serviço de urgência do Centro Hospitalar no dia 5 de novembro de 2017 com crise hipertensiva acompanhada de quadro de cefaleias, vómitos, prostração e diminuição da força muscular nos segmentos corporais do hemicorpo esquerdo, sendo referenciado através da via verde AVC. Posteriormente foi encaminhado para a Unidade de AVC do centro Hospitalar, após realização de Tomografia Axial Computorizada Crânio-Encefálica, compatível com lesão isquémica vertebro-basilar aguda.

$0 \mathrm{Sr}$. A apresentava como antecedentes pessoais hipertensão arterial e dislipidémia, apresentando como terapêutica habitual: captopril 12,5mg/dia; bisoprolol $2,5 \mathrm{mg} / \mathrm{dia}$; pravastatina $20 \mathrm{mg} / \mathrm{dia}$. Este é o seu primeiro internamento e desconhece qualquer tipo de alergias;

Quanto ao estado nutricional, o Sr. A apresentava um peso adequado para a sua altura (peso $82 \mathrm{~kg}$ e altura $180 \mathrm{~cm})$, correspondendo a um índice de massa corporal de $25,31 \mathrm{~kg} / \mathrm{m}^{2}$, valor que se manteve ao longo do programa de reeducação funcional da deglutição. Contudo, o Sr. A realizou 3 a 4 refeições por dia ao longo do internamento, apresentando alterações na segurança e efetividade na deglutição de alimentos com consistência líquida desde o dia da admissão, sendo evidente o risco de desidratação.

Desde o dia da admissão que o Sr. A tinha alteração na comunicação, nomeadamente na fluência do discurso (disartria). Não tinha alterações na audição ou visão, e não necessitava de nenhum mecanismo de compensação. A orientação alopsíquica e autopsíquica foram sempre uma constante no Sr. A. Apresentou perfeita consciência da sua situação clínica e demonstrou-se sempre participativo no programa instituído.
No âmbito das atividades de vida diária e das atividades instrumentais da vida diária, o Sr. A apresentava-se independente até ao internamento e refere ter uma vida social ativa.

Quanto à situação sociofamiliar e habitacional, o Sr. A referiu não ter dificuldades económicas. Vivia com a esposa numa casa térrea, no centro de uma localidade. A presença da esposa foi uma constante a partir do décimo dia de internamento, acompanhado, desde então, a sua evolução clínica e participou ativamente no programa instituído.

\section{Avaliação de Enfermagem de Reabilitação}

Os dados obtidos através da anamnese complementam os alcançados no exame físico da pessoa e orientam o enfermeiro de reabilitação na implementação de um programa baseado em diagnósticos e intervenções de enfermagem de reabilitação.

O programa de reabilitação e primeiro contato com o Sr. A teve início às 24 horas após o seu internamento no serviço, no dia 6 de novembro de 2017, e as intervenções de enfermagem de reabilitação foram implementadas até ao dia 24 de novembro de 2017. O Sr. A teve alta clínica no dia 28 de novembro de 2017.

No exame físico do Sr. A foi fundamental o recurso aos instrumentos de avaliação anteriormente mencionados para uma adequada categorização do grau de alteração da deglutição. 0 recurso a esses instrumentos de avaliação permitiu definir os diagnósticos de enfermagem de reabilitação e determinar os ganhos sensíveis aos cuidados de enfermagem de reabilitação.

De acordo com as fontes bibliográficas consultadas é defendido um intervalo de 48 em 48 horas para a avaliação com o EAT-10(3). Quanto à FOIS é sugerida a sua aplicação nas 24 horas após a admissão e 3 após uma avaliação inicial(5). Assim, o Sr. A foi sujeito a uma avaliação no início do programa de reeducação funcional da deglutição e manteve-se a periodicidade de avaliações num intervalo de três sessões, de forma a manter o tempo preconizado para a aplicação da escala de FOIS.

Ao ser identificada a alteração na deglutição do Sr. A com o score de EAT-10 e o nível de FOIS obtido na avaliação subjetiva, procedeu-se à avaliação objetiva com a aplicação do método de exploração clínico VVST.

Esta avaliação objetiva permitiu determinar a consistência e o volume dos alimentos para uma deglutição segura, confirmar ou corroborar o nível de FOIS obtido na avaliação subjetiva e fundamentar a dieta recomendada e supervisão necessária. Os sinais de alteração da segurança e efetividade na deglutição, detetados na realização do V-VST, foram registados num instrumento de registo desenvolvido para o efeito.

Em cada uma das avaliações efetuadas, para além de aplicados os instrumentos de avaliação acima mencionados, foram ajustados os diagnósticos de enfermagem e respetivas intervenções subordinados ao foco "deglutição". 
Visando uma abordagem holística, no decorrer do programa de reeducação funcional da deglutição, foi possível a colheita de outros dados que permitiram interpretar a condição ventilatória e o estado nutricional da pessoa. No que respeita à condição ventilatória, foi monitorizada e registada diariamente a saturação de oxigénio, obtida através da oximetria periférica. No âmbito da avaliação do estado nutricional foi monitorizado o Índice de Massa Corporal (IMC) da pessoa a cada seis sessões.

\section{Diagnósticos de Enfermagem de Reabilitação}

Os três diagnósticos de enfermagem que sustentaram o programa de reeducação funcional da deglutição foram definidos respeitando a linguagem da Classificação Internacional para a Prática de Enfermagem (CIPE $\left.{ }^{\circledR}\right)$ versão $2015^{(16)}$ e centraram-se no foco "Deglutição" e nas dimensões do "Conhecimento" e "Aprendizagem de Capacidades"(12). São eles:

1. Deglutição comprometida;

2. Potencial para melhorar conhecimento sobre exercícios e técnicas de deglutição;

3. Potencial para melhorar a capacidade para executar exercícios e técnicas de deglutição.

\section{Programa de Reeducação Funcional da Deglutição}

Ao longo do programa de reeducação funcional da deglutição, ao reunir os dados colhidos na avaliação subjetiva e objetiva do Sr. A, e após levantamento dos diagnósticos de enfermagem, foram propostas diversas intervenções de enfermagem (quadro 1).

\begin{tabular}{|c|c|c|}
\hline \multicolumn{3}{|c|}{ Foco Deglutição } \\
\hline Diagnóstico ativo & Enunciados de diagnóstico & $\begin{array}{c}\begin{array}{c}\text { Enunciados de ação de diagnóstico e de intervenções de } \\
\text { enfermagem }\end{array} \\
\end{array}$ \\
\hline \multirow{7}{*}{ Sessão 1 a 3} & \multirow{7}{*}{ Deglutição comprometida em grau moderado } & Avaliar capacidade de deglutição \\
\hline & & Monitorizar deglutição (EAT-10, V-VST, FOIS) \\
\hline & & Supervisionar a deglutição \\
\hline & & Incentivar deglutição \\
\hline & & Gerir dieta \\
\hline & & Planear dieta \\
\hline & & Posicionar a Pessoa \\
\hline \multirow{6}{*}{ Sessão 4 a 10} & \multirow{6}{*}{ Deglutição comprometida em grau reduzido } & Avaliar capacidade de deglutição \\
\hline & & Monitorizar deglutição (EAT-10, V-VST, FOIS) \\
\hline & & Supervisionar a deglutição \\
\hline & & Gerir dieta \\
\hline & & Planear dieta \\
\hline & & Posicionar Pessoa \\
\hline & \multicolumn{2}{|c|}{ Dimensão do Conhecimento } \\
\hline Diagnóstico ativo & Enunciado de diagnóstico & $\begin{array}{c}\begin{array}{c}\text { Enunciados de ação de diagnóstico e de intervenções de } \\
\text { enfermagem }\end{array}\end{array}$ \\
\hline \multirow{5}{*}{ Sessão 1 a 10} & \multirow{5}{*}{$\begin{array}{l}\text { Potencial para melhorar conhecimento sobre } \\
\text { exercícios e técnicas de deglutição }\end{array}$} & Ensinar sobre processo patológico \\
\hline & & Ensinar sobre complicações do processo patológico \\
\hline & & Ensinar sobre dieta (dieta adaptada) \\
\hline & & Ensinar sobre exercícios e técnicas de deglutição \\
\hline & & $\begin{array}{l}\text { Avaliar conhecimento sobre exercícios e técnicas de } \\
\text { deglutição }\end{array}$ \\
\hline & \multicolumn{2}{|c|}{ Dimensão da Aprendizagem de Capacidades } \\
\hline Diagnóstico ativo & Enunciado de diagnóstico & $\begin{array}{c}\begin{array}{c}\text { Enunciados de ação de diagnóstico e de intervenções de } \\
\text { enfermagem }\end{array}\end{array}$ \\
\hline \multirow[b]{2}{*}{ Sessão 1 a 10} & \multirow{2}{*}{$\begin{array}{l}\text { Potencial para melhorar capacidade para } \\
\text { executar exercícios e técnicas de deglutição }\end{array}$} & Instruir/Treinar exercícios e técnicas de deglutição \\
\hline & & $\begin{array}{l}\text { Avaliar capacidade para executar exercícios e técnicas de } \\
\text { deglutição }\end{array}$ \\
\hline
\end{tabular}

Quadro 1: Programa de Reabilitação Funcional da Deglutição(12;16)

Na aplicação do método de exploração clinico V-VST foram observadas manifestações clinicas nas diversas fases da deglutição, tal como relatadas na bibliografia consultada $^{(17,18)}$.

Tal facto possibilitou a identificação da fase da deglutição com maior número de alterações e orientou para a seleção do conjunto de exercícios e técnicas mais adequados para combater tais disfunções.

Os programas propostos apresentaram sempre as mesmas três componentes de treino: exercícios de amplitude de movimentos e fortalecimento muscular, treino de posturas compensatórias e treino de técnicas compensatórias da deglutição, tal como sugerido por 
diversos autores ${ }^{(19 ; 20 ;}$ 21). Foram definidos dois programas de reeducação funcional da deglutição a serem aplicados ao longo da abordagem ao Sr. A (quadro 2).

\begin{tabular}{|c|c|c|c|}
\hline $\begin{array}{c}\text { Fase da } \\
\text { deglutição } \\
\text { com maior } \\
\text { número de } \\
\text { alterações }\end{array}$ & Programa & Exercícios & $\begin{array}{l}\text { Duraç. } \\
\text { (min.) }\end{array}$ \\
\hline \multirow{3}{*}{$\begin{array}{c}\text { Fase oral } \\
\text { (Programa 1) }\end{array}$} & $\begin{array}{l}\text { Exercícios de } \\
\text { Fortalecimento } \\
\text { Muscular }\end{array}$ & $\begin{array}{l}\text { Lábios; } \\
\text { Língua; } \\
\text { Bochechas; } \\
\text { Língua e Bochechas; } \\
\text { Maxilar Inferior. }\end{array}$ & $15^{\prime}$ \\
\hline & $\begin{array}{l}\text { Posturas } \\
\text { compensatórias }\end{array}$ & $\begin{array}{l}\text { Posição Reclinada; } \\
\text { Extensão cervical; } \\
\text { Rotação cervical para o } \\
\text { lado menos funcional; } \\
\text { Rotação cervical para o } \\
\text { lado menos funcional e } \\
\text { extensão cervical; } \\
\text { Decúbito lateral com } \\
\text { cabeça apoiada. }\end{array}$ & $10^{\prime}$ \\
\hline & $\begin{array}{l}\text { Técnicas } \\
\text { compensatórias } \\
\text { da deglutição }\end{array}$ & $\begin{array}{l}\text { Controlo do bolo } \\
\text { alimentar; } \\
\text { Deglutição múltipla; } \\
\text { Deglutição de esforço. }\end{array}$ & 5 ' \\
\hline \multirow[b]{3}{*}{$\begin{array}{l}\text { Fase faríngea } \\
\text { (Programa 2) }\end{array}$} & $\begin{array}{l}\text { Exercícios de } \\
\text { Fortalecimento } \\
\text { Muscular }\end{array}$ & $\begin{array}{l}\text { Língua e bochechas; } \\
\text { Maxilar inferior; } \\
\text { Larínge. }\end{array}$ & $5^{\prime}$ \\
\hline & $\begin{array}{l}\text { Posturas } \\
\text { compensatórias }\end{array}$ & $\begin{array}{l}\text { Posição Reclinada; } \\
\text { Flexão Cervical; } \\
\text { Inclinação Cervical. }\end{array}$ & $5^{\prime}$ \\
\hline & $\begin{array}{l}\text { Técnicas } \\
\text { compensatórias } \\
\text { da deglutição }\end{array}$ & $\begin{array}{l}\text { Estimulação térmica; } \\
\text { Deglutição de esforço; } \\
\text { Deglutição supraglótica; } \\
\text { Deglutição super- } \\
\text { supraglótica; } \\
\text { Manobra de Mendelsohn; } \\
\text { Manobra de Masako; } \\
\text { Exercício de Shaker. }\end{array}$ & $20^{\prime}$ \\
\hline
\end{tabular}

Quadro 2: Exercícios e técnicas para a reeducação funcional da deglutição $(19 ; 20 ; 21)$.

Cada sessão de treino teve uma duração média de 30 minutos, de acordo com as evidencias detectadas na bibliografia consultada(19). A par dessas sessões, tal como mencionado anteriormente, foi implementado um plano de cuidados de enfermagem de reabilitação.

O plano de cuidados instituído apresentou-se mutável ao longo do programa de reeducação funcional da deglutição e, tendo em conta a abordagem holística característica deste estudo, não vigoraram apenas diagnósticos centrados no foco “Deglutição".

Foram enunciados diagnósticos de enfermagem como: Autocuidado: Beber comprometido; Alimentar-se dependente; Limpeza das vias aéreas comprometida; Risco de aspiração; Risco de desidratação; Risco de compromisso na ingestão nutricional; Défice sensorial presente; Equilíbrio corporal comprometido; Movimento muscular diminuído ${ }^{(12,16)}$.

As intervenções de enfermagem foram asseguradas em todas as sessões, visando a recuperação da função da deglutição (documentada pela evolução na escala de FOIS) assim como a reabilitação da pessoa no seu todo.

\section{RESULTADOS}

Como mencionado anteriormente, o Sr. A integrou um amplo projeto, tendo iniciado o programa de reeducação funcional da deglutição no início do período de seleção do projeto.

Após assinar o consentimento informado, cumpriu um total de 10 sessões, tendo sido realizadas 4 avaliações ao todo, respeitando o intervalo de 3 sessões, e manteve-se no programa até à recuperação funcional da deglutição.

Níveis diminuídos de consciência, a presença de fadiga e alterações neurológicas podem comprometer a resposta da pessoa e a manutenção do estado de alerta suficiente para favorecer a sua participação nas sessões terapêuticas ${ }^{(22)}$. Assim, antes de qualquer intervenção, foi sempre avaliado o nível de consciência através da aplicação da Escala de Comas de Glasgow.

Tal como mencionado anteriormente, pôde observar-se que o Sr. A permaneceu sempre vígil e colaborante, apresentando 15 pontos na referida escala, quer na avaliação inicial, quer nas restantes avaliações.

Seguiu-se a etapa da avaliação da percepção das alterações da deglutição, recorrendo à aplicação do questionário EAT-10. O questionário foi sempre preenchido pelo sr. A, apenas sendo necessária orientação para o seu preenchimento no dia da avaliação inicial.

Verifica-se, na análise das respostas obtidas nos questionários EAT-10, que os problemas percepcionados com maior frequência residiam em "engolir líquidos exige um esforço maior" e "eu tusso quando como", sendo relatados em todas as avaliações efetuadas. Tal facto sugere serem estes os problemas que mais inquietaram o Sr. A ao longo do programa de reeducação funcional da deglutição (gráfico 1 ).

$\mathrm{Na}$ análise estatística dos questionários EAT-10 também se verificou que, embora não fosse relatada em todas as avaliações, a dificuldade em deglutir comprimidos também foi um problema de relevo relatado pelo Sr. A (tabela 1).

$\mathrm{Na}$ primeira avaliação foi evidente a percepção da alteração na deglutição, sendo obtido uma pontuação final bastante elevada (13 pontos). Contudo, no final do programa de reeducação funcional da deglutição, a pontuação obtida foi inferior a 3 pontos (2 pontos) (tabela 2), sugerindo remissão total dos sintomas da deglutição comprometida ${ }^{(3,5)}$. 


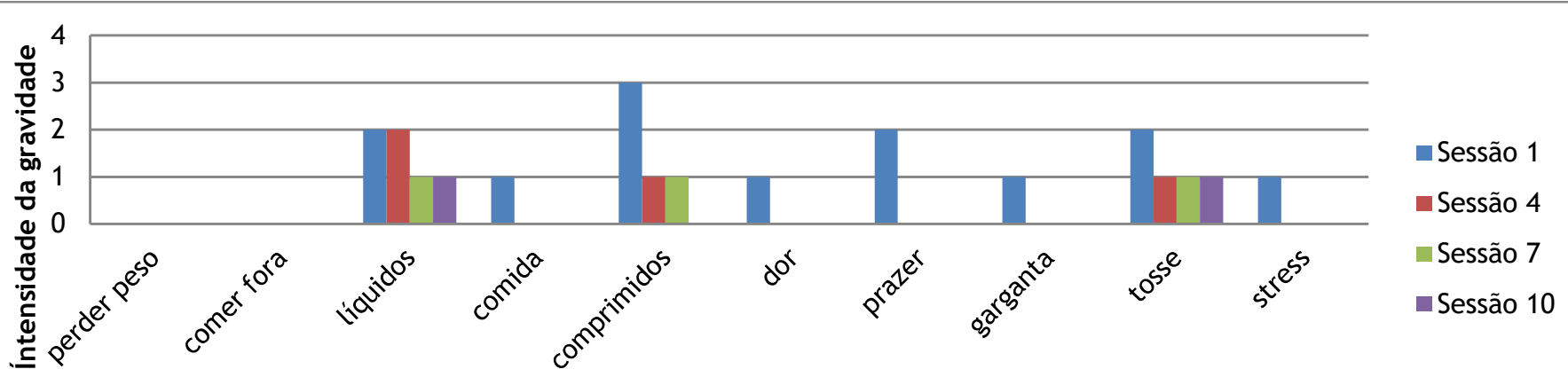

Problemas

Gráfico 1: Resultados dos questionários EAT-10 Fonte: Dados próprios

\begin{tabular}{|c|c|c|c|c|c|c|c|c|c|c|}
\hline & \multicolumn{10}{c|}{ Questão - Termos-Chave } \\
\hline $\begin{array}{c}\text { Dado } \\
\text { Estatístico }\end{array}$ & $\begin{array}{c}\text { Perder } \\
\text { peso }\end{array}$ & $\begin{array}{c}\text { Comer } \\
\text { fora }\end{array}$ & Líquidos & Comida & Comprimidos & Dor & Prazer & Garganta & Tosse & Stress \\
\hline Média & 0 & 0 & 1,5 & 0,25 & 1,25 & 0,25 & 0,5 & 0,25 & 1,25 & 0,25 \\
\hline Mínimo & 0 & 0 & 1 & 0 & 0 & 0 & 0 & 0 & 1 & 0 \\
\hline Máximo & 0 & 0 & 2 & 1 & 3 & 1 & 2 & 1 & 2 & 1 \\
\hline
\end{tabular}

Tabela 1: Análise estatística dos questionários EAT-10 Fonte: Dados próprios

\begin{tabular}{|c|c|}
\hline Sessão & Score EAT-10 \\
\hline 1 & 13 \\
\hline 4 & 4 \\
\hline 7 & 3 \\
\hline 10 & 2 \\
\hline
\end{tabular}

Tabela 2: Score total EAT-10 Fonte: Dados próprios

Relativamente à exploração clínica da deglutição, através da aplicação do método V-VST, verificou-se que, na sétima sessão, o Sr. A já tolerava bolus de líquido, mantendo sinais de alteração na segurança da deglutição em volumes altos $(20 \mathrm{ml})$. Aconselhou-se a ingestão de líquidos com supervisão e em médios volumes $(10 \mathrm{ml})$ (tabela 3$)$.

Na décima sessão o Sr. A já ingeria volumes elevados de líquidos $(20 \mathrm{ml})$ sem apresentar sinais de compromisso na segurança ou efetividade da deglutição. Constatouse que o Sr. A concluiu o programa de reeducação funcional da deglutição sem apresentar dessaturação de oxigénio, situação que era frequente no início (tabela 3).

\begin{tabular}{|c|c|c|c|c|}
\hline \multirow{2}{*}{ 悹 } & \multicolumn{2}{|c|}{$\begin{array}{c}\text { Último bolus com } \\
\text { sinais de segurança }\end{array}$} & \multicolumn{2}{|c|}{ Sinais clínicos observados } \\
\hline & $\begin{array}{l}\text { Consis- } \\
\text { tência }\end{array}$ & Volume & Segurança & Eficácia \\
\hline 1 & Néctar & $10 \mathrm{ml}$ & \begin{tabular}{|l|} 
Tosse; \\
Dessaturação \\
de oxigénio \\
$(4 \%)$
\end{tabular} & $\begin{array}{l}\text { Encerramento labial } \\
\text { ineficaz; } \\
\text { Resíduos orais; } \\
\text { Deglutições Múltiplas; } \\
\text { Resíduos faríngeos. }\end{array}$ \\
\hline 4 & Líquido & $5 \mathrm{ml}$ & Tosse & -- \\
\hline 7 & Líquido & $10 \mathrm{ml}$ & Tosse & -- \\
\hline 10 & Liquido & $20 \mathrm{ml}$ & -- & -- \\
\hline
\end{tabular}

Tabela 3: Síntese dos resultados do V-VST Fonte: Dados próprios
Os dados obtidos na aplicação do método de exploração clínico V-VST orientaram para a seleção dos exercícios e técnicas mais adequados.

$\mathrm{Na}$ sessão inicial foi selecionado o programa 1 e nas restantes sessões foi selecionado o programa 2 . $\mathrm{Na}$ sessão final, embora não existindo alterações no V-VST, aproveitou-se para reforçar as técnicas ministradas, selecionando-se o programa 2 . Observou-se que o Sr. A apresentou maior número de alterações na fase faríngea, o que justificou a seleção do programa $2 \mathrm{com}$ maior frequência.

Foram assegurados os cuidados especializados de enfermagem de reabilitação inerentes à pessoa com deglutição comprometida, direcionados às alterações apresentadas pelo $\mathrm{Sr}$. A, visando alcançar a sua máxima funcionalidade e autonomia.

Assim, relativamente ao diagnóstico "deglutição comprometida", observou-se uma progressão da autonomia do Sr. A no grau de compromisso ao longo do programa. No âmbito das dimensões do "Conhecimento" e "Aprendizagem de Capacidades", a mudança do programa de exercícios e técnicas da deglutição, do programa 1 para o programa 2 contribuiu para que existissem algumas lacunas na fase de transição. Contudo, essas lacunas foram sendo colmatadas (tabela 4).

Salienta-se a elevada adesão aos exercícios e técnicas de deglutição propostos.

O Sr. A demonstrou uma capacidade acrescida nos exercícios e técnicas que se realizaram com maior frequência ao longo do programa de reeducação funcional da deglutição, sendo observada maior dificuldade na realização da manobra de Masako (por ser uma técnica de difícil execução). A técnica de estimulação térmica foi, inicialmente, procedida pelo enfermeiro. Contudo, no final do programa, o Sr. A já demonstrou capacidade para a realizar. 


\begin{tabular}{|c|c|c|c|c|}
\hline Enunciado de diagnóstico & \multicolumn{4}{|c|}{ Sessões } \\
\hline \multirow{2}{*}{ Deglutição comprometida } & 1 & 4 & 7 & 10 \\
\hline & & & & -- \\
\hline \multicolumn{5}{|c|}{ Dimensão do Conhecimento } \\
\hline Enunciado de diagnóstico & \multicolumn{4}{|c|}{ Sessões } \\
\hline \multirow{2}{*}{$\begin{array}{l}\text { Potencial para melhorar conhecimento sobre exercícios e técnicas de } \\
\text { deglutição }\end{array}$} & 1 & 4 & 7 & 10 \\
\hline & & & & \\
\hline \multicolumn{5}{|c|}{ Dimensão da Aprendizagem de Capacidades } \\
\hline Enunciado de diagnóstico & \multicolumn{4}{|c|}{ Sessões } \\
\hline \multirow{2}{*}{$\begin{array}{l}\text { Potencial para melhorar capacidade para executar exercícios e técnicas } \\
\text { de deglutição }\end{array}$} & 1 & 4 & 7 & 10 \\
\hline & & & & \\
\hline
\end{tabular}

Legenda: grau moderado $\bigcirc$ grau reduzido $\bigcirc$ não demonstra $\bigcirc$ demonstra

Tabela 4: Evolução nos diagnósticos de enfermagem relacionados com o foco "Deglutição" Fonte: Dados próprios

Verificou-se um maior défice de conhecimento sobre a técnica de deglutição super-supraglótica. Embora a manobra de Masako seja a técnica onde a pessoa demonstrou maior dificuldade na execução, tal não se verificou com o conhecimento sobre a mesma. O Sr. A demonstrou um conhecimento mais consistente nos exercícios terapêuticos ministrados desde o início do programa.

O cuidador familiar (esposa) nem sempre esteve presente em todas as sessões, mas apreendeu de forma correta as atividades e técnicas abordadas ao longo do programa. Tal como o Sr. A, demonstrou conhecimento consistente nas técnicas que foram executadas com maior frequência ao longo do programa. Tanto o Sr. A como o cuidador familiar adquiriram, de forma gradual, conhecimentos sobre a técnica de consistência adaptada (utilização de espessante).

Tendo em conta a alteração na deglutição era evidente o "risco de compromisso na ingestão nutricional"(16). No final do programa, altura em que o Sr. A já apresentava capacidade funcional para ingerir refeições com duas ou mais consistências, tal risco deixou de estar presente. Foi monitorizado o IMC semanalmente e, tal como referido anteriormente, não foram detetadas alterações ao longo do programa.

$\mathrm{Na}$ análise dos dados obtidos constatou-se que o Sr. A apresentou melhoria funcional da deglutição, comprovada pela progressão no nível de FOIS. De salientar que o sr. A reverteu por completo as alterações na deglutição, atingindo o nível 7 na escala de FOIS (tabela 5).

\begin{tabular}{|c|c|}
\hline Sessão & Nível FOIS \\
\hline 1 & 4 \\
\hline 4 & 5 \\
\hline 7 & 6 \\
\hline 10 & 7 \\
\hline
\end{tabular}

Tabela 5: Síntese da categorização na escala de FOIS

Fonte: Dados próprios

\section{DISCUSSÃO}

A abordagem apresentada e os resultados alcançados demonstram que os cuidados especializados de enfermagem de reabilitação podem desempenhar um papel importante na intervenção precoce junto das pessoas com deglutição comprometida e na sua reeducação funcional.

Os enfermeiros são os profissionais que permanecem mais tempo junto das pessoas, sendo responsáveis por assegurar a sua autonomia e segurança ${ }^{(2)}$. Preconiza-se que, para uma abordagem eficaz à pessoa com deglutição comprometida, se considere a necessidade de encarar esta realidade como uma prioridade de atuação multidisciplinar ${ }^{(2,23)}$.

A realização de estudos nesta área é fundamental para estabelecer a eficácia da reabilitação em pessoas com alterações específicas da deglutição, definir o tratamento adequado para rentabilizar os custos e produzir resultados positivos ${ }^{(8)}$. Concorrendo para alcançar tal objetivo, o caso apresentado contemplou a avaliação da deglutição através da aplicação de instrumentos de avaliação válidos, fiáveis, responsivos e eficientes ${ }^{(24)}$ e da implementação de um programa de optimização da deglutição em função das alterações detetadas. Este conjunto de intervenções objetivou a recuperação funcional da deglutição da pessoa estudada.

$\mathrm{Na}$ seleção do conjunto de exercícios e técnicas da deglutição mais apropriado, o recurso aos instrumentos EAT-10 e V-VST foi fundamental para caracterizar as alterações observadas ${ }^{(3,4)}$.

A presença da alteração na deglutição pode conduzir a sentimentos de depressão e ansiedade durante as refeições ${ }^{(3)}$. A aplicação da ferramenta EAT-10 permitiu mensurar o grau de severidade dos sintomas, monitorizar a eficácia do programa instituído ${ }^{(3,5)} \mathrm{e}$ detetar os problemas percecionados com maior frequência e que mais inquietaram a pessoa abordada. Constatou-se uma redução significativa dos sintomas relatados.

A conjugação da ferramenta EAT-10 com o método de exploração clinico V-VST apresenta grande precisão no que concerne à deteção das alterações da deglutição( ${ }^{(4)}$. Os resultados apresentados com a aplicação destes instrumentos evocam a necessidade da realização de um rastreio clínico e treino de habilidades por alguém com competências na avaliação da deglutição, capaz de 
determinar a presença, gravidade e mecanismo da alteração $^{(7,23)}$.

O V-VST permitiu detetar alterações na segurança e efetividade da deglutição e forneceu os dados necessários para demonstrar que, no final do programa, a pessoa estudada já tolerava elevados volumes de líquidos, o que sugere a efetividade das intervenções planeadas.

De acordo com as manifestações clínicas observadas foi fundamental o ajuste dos exercícios de fortalecimento muscular, posturas compensatórias e técnicas facilitadoras da deglutição(19) ao longo desta abordagem. Tal ajuste permitiu a individualização do programa de reeducação funcional da deglutição.

A reeducação funcional da deglutição da pessoa com AVC deve incluir medidas terapêuticas centradas no aumento da sensibilidade oral, exercícios motores orais e manobras compensatórias de forma a prevenir a aspiração ${ }^{(25)}$. Tais aspetos foram sempre abordados nos dois programas de reabilitação propostos.

0 reforço positivo foi fundamental e necessário para o alcance dos objetivos delineados para o programa de reeducação funcional(26) da deglutição. No caso analisado, a pessoa demonstrou, de uma forma geral, deter conhecimento e capacidade para executar exercícios e técnicas de deglutição, sendo o reforço positivo a chave de tal sucesso.

A dificuldade da pessoa em realizar determinados exercícios terapêuticos, principalmente no âmbito técnicas compensatórias da deglutição, prendeu-se com a presença de défices motores e sensoriais que impossibilitaram a sua independência na realização das mesmas.

$\mathrm{Na}$ concretização do autocuidado e do autocontrolo é necessária uma abordagem estruturada dos cuidados de enfermagem de reabilitação, envolvendo os cuidadores familiares e informais nas intervenções propostas ${ }^{(27)}$. Assim, a participação do cuidador informal no processo de reabilitação da pessoa demonstrou-se fundamental, reforçando o seu papel na continuidade e reforço dos cuidados prestados.

Visando promover os processos de readaptação e promover a capacidade para o autocuidado (28), salienta-se a importância do planeamento dos cuidados de enfermagem de reabilitação baseados em diagnósticos de enfermagem em conformidade com as alterações observadas.

A efetividade do método de assistência defendida por Dorothea Orem depende, em grande parte, da criatividade do enfermeiro, da sua apreciação, conhecimento acerca da pessoa e respeito pela mesma. Um ambiente conducente ao desenvolvimento é igualmente um ambiente favorecedor da aprendizagem. ${ }^{(29)}$ A abordagem explanada neste estudo enquadra-se na teoria do défice de autocuidado de Dorothea Orem que foca o papel da Enfermagem na aprendizagem e desenvolvimento de capacidades da pessoa cuidada.

O raciocínio clínico que o enfermeiro faz culmina num diagnóstico e envolve tomar decisões com base na identificação das condições clínicas da pessoa (critérios de diagnóstico), neste sentido, quanto maior for a acurácia de um diagnóstico, mais preciso deverá ser o processo de decisão clínica(30). A utilização dos instrumentos que permitiram avaliar as alterações da deglutição contribuiu para a definição de critérios de diagnóstico de enfermagem de reabilitação.

Por outro lado, estes instrumentos ajudaram a avaliar a progressão do estado da pessoa com a intervenção do Enfermeiro de Reabilitação e, conjuntamente com as intervenções implementadas, concorreram para a reeducação funcional da deglutição, sendo tal finalidade atestada pela progressão na escala FOIS.

O estudo deste caso permitiu refletir sobre a relevância da avaliação sistemática da deglutição e reforçar a ideia de que o risco nutricional da pessoa com AVC exige um acompanhamento e esforço contínuos ${ }^{(31)}$.

Dos resultados da implementação deste programa sobressaem algumas limitações. Seria fundamental, numa futura pesquisa, alargar o tempo disponível para a implementação das diversas atividades terapêuticas, permitindo obter resultados mais consistentes. De igual modo, o facto de apenas ser abordado o caso de uma pessoa, impossibilita a realização de inferências sobre os resultados obtidos.

Não existem muitas publicações por enfermeiros, na área da reeducação funcional da deglutição. Desta forma, a presente exposição poderá dar visibilidade às intervenções dos enfermeiros de reabilitação junto da pessoa com esta alteração. Assim, torna-se emergente a necessidade de realização de mais estudos subordinados a esta temática, nomeadamente estudos experimentais ou quasi-experimentais.

O caso apresentado poderá contribuir para uma prática de enfermagem especializada devidamente sistematizada, possibilitando a disseminação do conhecimento acerca da intervenção do enfermeiro de reabilitação junto da pessoa com deglutição comprometida, com vista a fortalecer o ensino e a prática desta especialidade.

\section{CONSIDERAÇÕES FINAIS}

O presente estudo de caso permitiu destacar a efetividade das intervenções de enfermagem de reabilitação no âmbito da promoção da segurança e funcionalidade da pessoa com deglutição comprometida, sendo esta uma possível abordagem a ter em conta em pessoas com alterações desta natureza.

Ao longo do programa implementado foi fundamental detectar a capacidade da pessoa para alimentar-se e hidratar-se de forma segura e eficaz, tal foi possível através da monitorização constante da consciência com recurso à Escala de Coma de Glasgow.

A avaliação do risco de alteração na deglutição foi possível com recurso ao questionário EAT-10, que permitiu identificar os sintomas da deglutição comprometida e o seu grau de severidade. 
Os sinais clínicos identificados com a aplicação do método de exploração clínico (V-VST) foram fundamentais para a seleção do conjunto de exercícios e adequado.

Os instrumentos de avaliação (FOIS, EAT-10 e V-VST) para além de ajudarem a definir os diagnósticos de enfermagem de reabilitação enunciados, tornaram mais objetivos os critérios de diagnóstico, facilitaram na implementação de enunciados de ação de diagnóstico e de intervenções de enfermagem adequados à situação clínica da pessoa e à disfunção observada, e permitiram uma abordagem holística e personalizada.

A evolução da pessoa abordada foi visível através dos resultados obtidos na aplicação dos instrumentos de avaliação e da progressão positiva no grau de compromisso nos diagnósticos de enfermagem enunciados. As intervenções de enfermagem demonstraram ter sido efetivas para reverter as alterações da deglutição e concorreram para melhorar a autonomia e independência da pessoa estudada.

Ao longo do programa foi evidente o risco de compromisso na ingestão nutricional, mas não foram observadas grandes oscilações nos valores de IMC que comprovassem tal compromisso.

\section{REFERÊNCIAS}

1. Durà Mata MJ, Molleda M, Sánchez-Migallón JM, Viña C, Pollán C, Calderón C. Integral management oropharyngeal dysphagia. Ann Phys Rehabil Med [Internet]. Elsevier BV; 2018 Jul; 61: e506-e507. Available from: http: //dx.doi.org/10.1016/j.rehab.2018.05.1179

2. Palli C, Fandler S, Doppelhofer K, Niederkorn K, Enzinger C, Vetta C, et al. Early Dysphagia Screening by Trained Nurses Reduces Pneumonia Rate in Stroke Patients. Stroke [Internet]. Ovid Technologies (Wolters Kluwer Health); 2017 Jul 17; 48(9): 25835. $\quad$ Available $\quad$ from: http://dx.doi.org/10.1161/strokeaha.117.018157

3. Nogueira DS, Ferreira PL, Reis EA, Lopes IS. Measuring Outcomes for Dysphagia: Validity and Reliability of the European Portuguese Eating Assessment Tool (P-EAT-10). Dysphagia [Internet]. Springer Nature; 2015 Jun 16; 30(5): 511-20. Available from: http://dx.doi.org/10.1007/s00455-015-9630-5

4. Rofes L, Arreola V, Mukherjee R, Clavé P. Sensitivity and specificity of the Eating Assessment Tool and the Volume-Viscosity Swallow Test for clinical evaluation of oropharyngeal dysphagia. Neurogastroenterol Motil [Internet]. Wiley; 2014; 26(9): 1256-65. Available from: http://dx.doi.org/10.1111/nmo.12382

5. Queirós, A., Moreira, S., Silva, A., Costa, R. Lains, J. Contributo para a Adaptação e Validação da Eat Assessment Tool (EAT-10) e da Functional Oral Intake Scale (FOIS). Rev Soc Port Med Fis Reabil [Internet], 2013; 24(2): 25-30. Available from: https://spmfrjournal.org/index.php/spmfr/article/view/108

6. Courey MS, Pletcher SD. Upper Airway Disorders. Clin Pulm Med [Internet]. Elsevier; 2016; 877-896.e5. Available from: http://dx.doi.org/10.1016/b978-1-4557-3383-5.00049-x

7. Braga, R. Reeducação da Deglutição. in C. Marques-Vieira; L. Sousa (Eds). Cuidados de Enfermagem de Reabilitação à Pessoa ao Longo da Vida. Loures: Lusodidacta; 2017 Jan: 263-70

8. Easterling C. 25 Years of Dysphagia Rehabilitation: What Have We Done, What are We Doing, and Where are We Going? Dysphagia [Internet]. Springer Nature; 2017 Jan 2; 32(1): 50-4. Available from: http://dx.doi.org/10.1007/s00455-016-9769-8

9. Ordem dos Enfermeiros. Guia Orientador de Boas Práticas Cuidados à pessoa com alterações da mobilidade posicionamentos, transferências e treino de deambulação. Lisboa: Ordem dos Enfermeiros; 2013. Available from: https://www.ordemenfermeiros.pt/arquivo/publicacoes/Docum ents/GOBP_Mobilidade_VF_site.pdf

10. Wade D. Rehabilitation - a new approach. Part two: the underlying theories. Clin Rehabil [Internet]. SAGE Publications; 2015 Nov 18;29(12):1145-54. Available from: http://dx.doi.org/10.1177/0269215515601175

11. Petronilho, F., Machado, M. Teorias de Enfermagem e Autocuidado: Contributos para a Construção do Cuidado de Reabilitação. in C. Marques-Vieira; L. Sousa (Eds), Cuidados de Enfermagem de Reabilitação à Pessoa ao Longo da Vida. Loures: Lusodidacta; 2017 Jan: 3-14.

12. Ordem dos Enfermeiros. Padrão Documental dos Cuidados de Enfermagem da Especialidade de Enfermagem de Reabilitação. Porto: Mesa do Colégio da Especialidade de Enfermagem de Reabilitação; $2015 . \quad$ Available from: https://www.ordemenfermeiros.pt/arquivo/colegios/Document s/2015/MCEER_Assembleia/PadraoDocumental_EER.pdf

13. Andrade SR de, Ruoff AB, Piccoli T, Schmitt MD, Ferreira A, Xavier ACA. $O$ estudo de caso como método de pesquisa em enfermagem: uma revisão integrativa. Texto \& contexto enferm. [Internet]. FapUNIFESP (SciELO); 2017 Nov 17;26(4). Available from: http://dx.doi.org/10.1590/0104-07072017005360016

14. Gagnier JJ, Riley D, Altman DG, Moher D, Sox H, et al. Case Reporting (CARE) Guidelines Checklist. PsycTESTS Dataset [Internet]. American Psychological Association (APA); 2013; Available from: http://dx.doi.org/10.1037/t29881-000

15. Ferreira, D., Santos, A. Avaliação da Pessoa com Patologia Respiratória. in C. Marques-Vieira; L. Sousa (Eds). Cuidados de Enfermagem de Reabilitação à Pessoa ao Longo da Vida. Loures: Lusodidacta; 2017 Jan: 167-80

16. Ordem dos Enfermeiros. Classificação Internacional para a Prática de Enfermagem Versão 2015. Lisboa: Ordem dos Enfermeiros; 2016 Available from: https://futurosenf.files.wordpress.com/2017/04/cipe_2015.pdf

17. Glenn-Molali, N. Alimentação e Deglutição. in Hoeman S. P. Enfermagem de Reabilitação: Prevenção, Intervenção e Resultados Esperados. 4. ed. Loures: Lusodidacta; 2011 Set: 295317

18. Ricci Maccarini A, Filippini A, Padovani D, Limarzi M, Loffredo M, Casolino D. Clinical non-instrumental evaluation of dysphagia. Acta otorhinolaryngol. ital..[Internet] Dec. 2007; 27(6): 299-305. Available https://www.ncbi.nlm.nih.gov/pmc/articles/PMC2640056/pdf/ 0392-100X.27.299.pdf

19. Alves ICF, Andrade CRF de. Mudança funcional no padrão de deglutição por meio da realização de exercícios orofaciais. CoDAS [Internet]. FapUNIFESP (SciELO); 2017; 29(3). Available from: http://dx.doi.org/10.1590/2317-1782/20172016088

20. Lazarus CL. History of the Use and Impact of Compensatory Strategies in Management of Swallowing Disorders. Dysphagia [Internet]. Springer Nature; 2017 Jan 28; 32(1): 3-10. Available from: http://dx.doi.org/10.1007/s00455-016-9779-6

21. Barkmeier-Kraemer JM, Clark HM. Speech-language pathology evaluation and management of hyperkinetic disorders affecting speech and swallowing function. Tremor Other Hyperkinet Mov. 2017; 7. Available from: http://dx.doi.org/10.7916/D8Z32B30

22. Thompson R. Managing dysphagia in patients with neurological conditions. British J Neurosci Nurs. [Internet]. Mark Allen Group; 2017 Jun 2;13(3):106-10. Available from: http://dx.doi.org/10.12968/bjnn.2017.13.3.106

23. European Society for Swallowing Disorders. Dysphagia [Internet]. Springer Nature; 2013 Apr 27; 28(2): 280-335. Available from: http://dx.doi.org/10.1007/s00455-013-9455-z

24. Sousa L, Marques-Vieira C, Severino S, Caldeira S. Propriedades psicométricas de instrumentos de avaliação para a investigação e prática dos enfermeiros de reabilitação. in C. Marques-Vieira; L. Sousa (Eds). Cuidados de Enfermagem de Reabilitação à Pessoa ao Longo da Vida. Loures: Lusodidacta; 2017 Jan:113-22.

25. Altman KW, Richards A, Goldberg L, Frucht S, McCabe DJ. Dysphagia in Stroke, Neurodegenerative Disease, and Advanced Dementia. Otolaryngol Clin North Am [Internet]. Elsevier BV; 2013 Dec; 46(6): 1137-49. Available from: http://dx.doi.org/10.1016/j.otc.2013.08.005

26. Li C-M, Wang T-G, Lee H-Y, Wang H-P, Hsieh S-H, Chou M, et al. Swallowing Training Combined With Game-Based Biofeedback in Poststroke Dysphagia. PM\&R [Internet]. Elsevier BV; 2016 Aug;8(8):773-9. Available from: http://dx.doi.org/10.1016/j.pmrj.2016.01.003 
27. Ferreira, F., Fonseca, C., Ramos, A., Lopes, M. J., Santos, V. Estudo de necessidades de cuidados de pessoas com 65 e mais anos de idade, proposta de intervenção dos cuidados de enfermagem de reabilitação. J Aging Innov [Internet]. 20176 (3): 32-42. Available from:

https://dspace.uevora.pt/rdpc/bitstream/10174/22855/1/4

Necessidades-de-cuidados-de-Enfermagem-de-

Reabilitac\%CC\%A7a\%CC\%830-proposta-de-

intervenc\%CC\%A7a\%CC\%83o.pdf

28. Regulamento $n^{\circ} 350 / 2015$ de 22 de junho da Ordem dos Enfermeiros. Diário da República. 2a'Série, 119; 2015. Available from:

http://www.ordemenfermeiros.pt/legislacao/Documents/Legisl acaoOE/RegulamentoPadQualidadeCuidEspecializEnfReabilitacao _DRJun2015.pdf
29. Orem, D. E. Nursing: Concepts of practice (6th ed.) St. Louis, MO: Mosby; 2001

30. Caldeira S, Chaves ED, Carvalho EC, Vieira MM. Validation of nursing diagnoses: the differential diagnostic validation model as a strategy. Rev Enferm UFPE [Internet]. 2012:1441-5. Available from: http: //dx.doi.org/10.5205/01012007

31. Kampman MT, Eltoft A, Karaliute M, Børvik MT, Nilssen $H$, Rasmussen I, et al. Full Implementation of Screening for Nutritional Risk and Dysphagia in an Acute Stroke Unit. Neurohospitalist [Internet]. SAGE Publications; 2015 Jun 30; 5(4): 205-11. Available from: http://dx.doi.org/10.1177/1941874415588749 